\title{
Analytically Explicit Results for the Distribution of the Number of Customers Served during a Busy Period for Special Cases of the $M / G / 1$ Queue
}

\author{
M. L. Chaudhry ${ }^{1}$ and Veena Goswami $\mathbb{D}^{2}$ \\ ${ }^{1}$ Department of Mathematics and Computer Science, Royal Military College of Canada, P.O. Box 17000, \\ Kingston, Ontario, K7K 7B4, Canada \\ ${ }^{2}$ School of Computer Applications, Kalinga Institute of Industrial Technology, Bhubaneswar 751 024, India \\ Correspondence should be addressed to Veena Goswami; veena_goswami@yahoo.com
}

Received 19 May 2019; Revised 27 July 2019; Accepted 1 August 2019; Published 27 August 2019

Academic Editor: Aera Thavaneswaran

Copyright (C) 2019 M. L. Chaudhry and Veena Goswami. This is an open access article distributed under the Creative Commons Attribution License, which permits unrestricted use, distribution, and reproduction in any medium, provided the original work is properly cited.

\begin{abstract}
This paper presents analytically explicit results for the distribution of the number of customers served during a busy period for special cases of the $M / G / 1$ queues when initiated with $m$ customers. The functional equation for the Laplace transform of the number of customers served during a busy period is widely known, but several researchers state that, in general, it is not easy to invert it except for some simple cases such as $M / M / 1$ and $M / D / 1$ queues. Using the Lagrange inversion theorem, we give an elegant solution to this equation. We obtain the distribution of the number of customers served during a busy period for various service-time distributions such as exponential, deterministic, Erlang- $k$, gamma, chi-square, inverse Gaussian, generalized Erlang, matrix exponential, hyperexponential, uniform, Coxian, phase-type, Markov-modulated Poisson process, and interrupted Poisson process. Further, we also provide computational results using our method. The derivations are very fast and robust due to the lucidity of the expressions.
\end{abstract}

\section{Introduction}

Emphasis on numerical transform inversion has become an important method for computing probability distributions in stochastic models [1-3]. In queueing theory, as transforms can often represent probability distributions, it is necessary to find the numerical inversion technique. Generally, the analytical inverse transform is difficult to evaluate or obtain in closed explicit form. For example, in an $M / G / 1$ queueing system, the probability generating function (PGF) of the distribution of the number of customers served during a busy period and the Laplace transform (LT) of the busy period distribution are represented as functional equations.

Queueing systems are directly applicable to many fields such as telecommunication, production, and computer systems. Most performance measures analyze the system to reduce the delays experienced by a customer. The analysis of the busy period is a significant component of any queueing system from the server's viewpoint, which helps in the efficient planning of the system. Understanding the characteristics of the busy period could manage to prevent server overload. When an arriving customer gets the system empty, a busy period begins and ends when the system next becomes empty.

In the case of $M / D / 1$ queue, Borel [4] first found the distribution of the number of customers served during the busy period when $m=1$ by a geometric argument, and Tanner [5] showed that Borel's argument is valid for general $m$, where $m$ is the number that initiates the busy period. Kendall [6] also obtained a continuous analogue of this distribution. Haight [7] studied the distribution in the case of $M / M / 1$ queue, analogous to the method used by BorelTanner. Using different approaches, the distribution of the number served during a busy period has been considered by 
Prabhu [8, 9], Haight [7, 10, 11], Takács [12, 13], Kemp and Kemp [14], and Feller [15]. Takács studied using a generalization of the classical ballot theorem [16]. Heyman [17] obtained an approximation for the busy period of the $M / G / 1$ queue using a diffusion model. The joint distribution of the length of the busy period and the number of customers served during that busy period for the $M / G / 1$ queues has been studied by Prabhu [9]. Consul and Shenton [18] showed that Lagrange distributions give a general form for the probability distribution of the number of customers served at a single counter in a busy period with the inputs following a discrete distribution and the service-time being constant.

For the queueing system $M / G / 1$, one of the important distributions is the number of customers served during a busy period. This distribution has been discussed by many researchers. In particular, see $[19,20,21]$ and references therein. Most of the researchers mentioned there either give only the functional equation or using that they give a few moments or solve that equation numerically (see, e.g., [21]). In general, as stated by them that getting an explicit form of this distribution being difficult, they give results only in some simple cases such as $M / M / 1$ and $M / D / 1$ (see, e.g., $[20,21])$. In this paper, for the queueing system $M / G / 1$, using the well-known Lagrange inversion theorem, we give explicit results for the distribution of the number of customers served during a busy period by considering many service-time distributions such as exponential, deterministic, Erlang- $k$, gamma, chi-square, inverse Gaussian, generalized Erlang, matrix exponential, hyperexponential, uniform, Coxian, phase-type, Markov-modulated Poisson process, and interrupted Poisson process. In addition, we also give numerical results. The benefit of these closed-form expressions is that they are comparatively simple, elegant, and can be easily computed numerically. The problem of finding the number of customers served during a busy period in various systems such as computer communication systems is useful in developing congestion control strategies.

The paper is organized as follows. In Section 2, we describe the model in detail, derive an equation for the Laplace transform of the number of customers served during a busy period, and find the moments of the number served during a busy period using functional equation. We also present an analytical derivation of the distribution of the number of customers served during a busy period for the $M / G / 1$ system using Lagrange inversion theorem. Section 3 gives analytically explicit results for the distribution of the number of customers served during a busy period by considering many service-time distributions. Section 4 provides computational results. Section 5 concludes the paper.

\section{2. $M / G / 1$ Queue}

In this section, we consider the distribution of the number of customers served during a busy period for the continuoustime $M / G / 1$ queueing system when initiated by $m$ customers. We assume that the interarrival times of customers are independent and follow an exponential distribution with common mean interarrival time $1 / \lambda$. The service-times are independent and identically distributed as a random variable
$S$ with a general distribution function $B(t)(t \geq 0)$, probability density function (pdf) $b(t)(t \geq 0)$, Laplace transform (LT) $\bar{b}(s)=\int_{0}^{\infty} e^{-s t} b(t) d t, s \geq 0$, and mean service-time $1 / \mu=-\bar{b}^{(1)}(0)$, where $\bar{b}^{(1)}(0)$ denotes the first derivative of $\bar{b}(s)$ evaluated at $s=0$. We assume that $\rho=(\lambda / \mu)<1$. For any integer $j \geq 0$, let $\alpha_{j}$ be the probability that $j$ customers arrive during the service period of a single customer. Let $\alpha(z)$ be the generating function of $\left\{\alpha_{j}\right\}$ and it is given by $\alpha(z)=\sum_{j=0}^{\infty} \alpha_{j} z^{j}$; that is,

$$
\begin{aligned}
\alpha(z) & =\sum_{j=0}^{\infty} \alpha_{j} z^{j}=\sum_{j=0}^{\infty} \int_{0}^{\infty} \frac{(\lambda t)^{j} e^{-\lambda t}}{j !} b(t) d t \\
& =\int_{0}^{\infty} e^{-\lambda t} \sum_{j=0}^{\infty} \frac{(\lambda t z)^{j}}{j !} b(t) d t \\
& =\bar{b}(\lambda-\lambda z) .
\end{aligned}
$$

In this paper, we assume that customers initiate a busy period when $m \geq 1$. The case $m>1$ can be considered as the initial busy period where a queue is permitted to build before the server starts working. The case $m=1$ presents subsequent busy periods where the arrival of a single customer terminates the idle period. We also assume that $\rho<1$, so that all busy periods will terminate with probability one. For the queueing system $M / G / 1$, one of the important distributions is the number of customers served during a busy period. Most of the researchers either give only the functional equation which we derive below for completeness sake or using that equation, they give a few moments or solve that equation numerically $[19,20,21]$.

\subsection{Functional Equation for the Number of Customers Served} during a Busy Period. We present the distribution of the number of customers served during a busy period for the continuous-time $M / G / 1$ queueing system. A busy period is initiated by a single customer. It begins at the instant of the arrival of a customer to an empty system and ends at the instant the server becomes idle for the first time. When the busy period ends, the idle period starts and ends when the next customer arrives. Let $N(T) \equiv N=$ be the number of customers served during a busy period $T$. Since the arrivals are Poisson distributed, it implies that the lengths of subbusy periods $T_{i}$ are independent and identically distributed random variables and equal to the distribution of the length of the total busy period $T$. The number of customers served during each busy period is independent and identically distributed and equal to the distribution of number of customers served during a complete busy period. Let $N_{i}, i=$ $1,2,3, \ldots, A$, be the number of customers served during the busy period generated by the $i$ th arrival, $A$ a random variable representing the number of arrivals that arrive during $S, N$ the number served during the entire busy period, and its probability generating function $R(z)=E\left(z^{N}\right)$. Then, since each arrival during the initial service-time of the busy period will generate its own distribution of the number served, we may write $N=1+\sum_{i=0}^{A} N_{i}$ with $N_{0}=0$. Now, since the 
arrivals follow a Poisson process, $N_{i}$ are independent of $A$ and $S$ and are also independent random variables identically distributed as $N$, we may write the following by conditioning $N$ by $A$ and $S$ :

$$
\begin{aligned}
R(z)= & \sum_{n=1}^{\infty} P(N=n) z^{n}=E\left(z^{N}\right)=E\left[E\left[z^{N} \mid A, S\right]\right] \\
= & \int_{0}^{\infty} \sum_{n=0}^{\infty} E\left[z^{1+\sum_{j=1}^{A} N_{j}} \mid A=n, S=t\right] \\
& \cdot P(A=n \mid S=t) b(t) d t \\
= & z \sum_{n=0}^{\infty}[R(z)]^{n} \int_{0}^{\infty} \frac{(\lambda t)^{n}}{n !} e^{-\lambda t} b(t) d t \\
= & z \int_{0}^{\infty} e^{-(\lambda-\lambda R(z)) t} b(t) d t \\
= & z \bar{b}(\lambda-\lambda R(z)),
\end{aligned}
$$

which shows that the probability generating function for the number of customers served during a busy period satisfies the above functional equation.

\subsection{Distribution of the Number of Customers Served during a} Busy Period for the $M / G / 1$ System. In this subsection, we present the derivation of the distribution of a number of customers served during a busy period for the $M / G / 1$ system using Lagrange inversion theorem. In mathematical analysis, the Lagrange inversion theorem provides the Taylor series expansion of the inverse of an analytic function. Closedform solutions of (2) can be found using Lagrange's inversion theorem. Applying (1) in (2), we may rewrite as

$$
R(z)=z \alpha(R(z)), \quad|z| \leq 1 .
$$

By Lagrange inversion theorem, we get

$$
R(z)=\sum_{n=1}^{\infty} \frac{z^{n}}{n !}\left\{D_{z}^{n-1} \alpha^{n}(z)\right\}_{z=0}
$$

where $D_{z}^{x} y(z)$ denotes the $x$ th derivative of $y(z)$ with respect to $z$ evaluated at $z=0$ and $D_{z}^{0} y(z)=y(z)$. For a particular service-time distribution $\bar{b}(s)$, we can easily get an expression for $\alpha(z)$ and thus an expression for $P(N=n)$. For more details, one may refer studies of Consul and Famoye [22] and Jain et al. [23]. Suppose that there are $m(\geq 1)$ customers at the beginning of the busy period $T$. Then, the PGF of the number $N_{m}(T)$ served during the busy period is given by $R^{m}(z)$, where $R(z)$ is the PGF of the number served during the busy period that begins by a single customer. The probability generating function of $N_{m}$ is $\sum_{n=m}^{\infty} P\left(N_{m}=n\right) z^{n}=R^{m}(z)$, and its Lagrange's series expansion simplifies to

$$
R^{m}(z)=\sum_{n=m}^{\infty} \frac{m z^{n}}{n(n-m) !}\left\{D_{z}^{n-m} \alpha^{n}(z)\right\}_{z=0} .
$$

This provides

$$
\begin{aligned}
P\left(N_{m}=n\right)=\frac{m}{n(n-m) !} & \left\{D_{z}^{n-m} \alpha^{n}(z)\right\}_{z=0}, \\
n & =m, m+1, m+2, \ldots .
\end{aligned}
$$

Equation (6) provides the distribution of a number of customers served during a busy period for the $M / G / 1$ system when initiated with $m$ customers. Taking into consideration various service-time distributions, we present the distribution of a number of customers served during a busy period in Section 3. In the derivation of analytical results, we also use Leibniz's rule. The general Leibniz's rule states that if $f(x)$ and $g(x)$ are $n$-times differentiable functions, then the product $f(x) g(x)$ is also $n$-times differentiable and its $n$th derivative is given by

$$
D_{x}^{n}(f(x) g(x))=\sum_{i=0}^{n}\left(\begin{array}{c}
n \\
i
\end{array}\right)\left\{D_{x}^{n-i} f(x)\right\}\left\{D_{x}^{i} g(x)\right\} .
$$

The formula can be generalized to the product of $k$ differentiable functions $f_{1}, f_{2}, \ldots, f_{k}$ :

$$
\begin{aligned}
D_{x}^{n}\left(f_{1}(x) f_{2}(x) \ldots f_{k}(x)\right)= & \sum_{i_{1}+i_{2}+\ldots+i_{k}=n}\left(\begin{array}{c}
n \\
i_{1}, i_{2}, \ldots, i_{k}
\end{array}\right) \\
& \cdot \prod_{1 \leq j \leq k} D_{x}^{i_{j}}\left(f_{j}\right),
\end{aligned}
$$

where the sum extends over all $k$-tuples $\left(i_{1}, i_{2}, \ldots, i_{k}\right)$ of nonnegative integers with $\sum_{j=1}^{k} i_{j}=n$ and $\left(\begin{array}{c}n \\ i_{1}, i_{2}, \ldots, i_{k}\end{array}\right)=$ $n ! / i_{1} ! i_{2} ! \ldots i_{k} !$ are the multinomial coefficients.

2.3. Moments of the Number Served during a Busy Period Using Functional Equation. Here, we obtain mean and variance of the number of customers served during a busy period for the queueing system $M / G / 1$. Let $v_{k}$ be the $k$ th moment of the service-time; that is, $v_{k}=E\left(S^{k}\right)=\left.(-1)^{k}\left(d^{k} \bar{b}(s) / d s^{k}\right)\right|_{s=0}$ and variance $\sigma^{2}=v_{2}-v_{1}^{2}$. Here, $\nu_{1}=1 / \mu$. Let $E\left(N^{r}\right), r=$ $1,2, \ldots$ be the $r$ th moment of the number served during a busy period when initiated by single customer. We obtain from (2) the following

$$
R^{(1)}(1)=\bar{b}(0)-\lambda \bar{b}^{(1)}(0) R^{(1)}(1)=\bar{b}(0)+\lambda \nu_{1} R^{(1)}(1),
$$

implying

$$
\begin{aligned}
R^{(1)}(1) \equiv & E(N)=\frac{1}{1-\lambda \nu_{1}}=\frac{1}{1-\rho} \\
R^{(2)}(1)= & -2 \lambda \bar{b}^{(1)}(0) R^{(1)}(1)+\bar{b}^{(2)}(0)\left[-\lambda R^{(1)}(1)\right]^{2} \\
& -\lambda \bar{b}^{(1)}(0) R^{(2)}(1) \\
= & 2 \frac{\lambda}{\mu} R^{(1)}(1)+\nu_{2}\left[-\lambda R^{(1)}(1)\right]^{2}+\frac{\lambda}{\mu} R^{(2)}(1),
\end{aligned}
$$


giving

$$
R^{(2)}(1)=\frac{2 \rho(1-\rho)+\lambda^{2} \nu_{2}}{(1-\rho)^{3}} .
$$

Since $R^{(2)}(1)=E\left(N^{2}\right)-E(N)$, we have

$$
E\left(N^{2}\right)=\frac{2 \rho(1-\rho)+\lambda^{2} \nu_{2}}{(1-\rho)^{3}}+\frac{1}{1-\rho} .
$$

The variance of the number of customers served during a busy period when initiated by single customer is

$$
\operatorname{Var}(N)=\frac{\rho(1-\rho)+\lambda^{2} \nu_{2}}{(1-\rho)^{3}}
$$

When a busy period begins with an initial number of $m$ customers in the system, the transition of the underlying Markov process from $m$ to 0 can be made up of $m$ intervals with the same distribution corresponding the transitions from $m \longrightarrow m-1, m-1 \longrightarrow m-2, \ldots, 1 \longrightarrow 0$ because of the Markovian property of the arrival process. These $m$ independent busy periods start with single customer in the system so that $N_{m}=\sum_{j=1}^{m} N_{1}(j)$. Hence, if $N_{m}$ is the random variable representing a busy period initiated by $m$ customers, we have

$$
\begin{aligned}
E\left(N_{m}\right) & =\sum_{j=1}^{m} E\left(N_{1}(j)\right)=m E\left(N_{1}\right)=\frac{m}{1-\rho}, \\
\operatorname{Var}\left(N_{m}\right) & =\frac{m\left(\rho(1-\rho)+\lambda^{2} v_{2}\right)}{(1-\rho)^{3}} .
\end{aligned}
$$

Note that higher order moments can be obtained similarly.

\section{Distributions}

In this section, we derive the distribution of the number of customers served during a busy period for different servicetime distributions. Using Lagrange's expansion, we find the number of customers served during a busy period when initiated with $m$ customers for the exponential, deterministic, gamma, chi-square, mixed generalized Erlang, matrix exponential, inverse Gaussian, hyperexponential, uniform, Coxian, phase-type, Markov-modulated Poisson process, and interrupted Poisson process service-time distributions. In the literature, explicit results are available only for exponential and deterministic service-time distributions.

3.1. Exponential Distribution. The exponential distribution has probability density function (pdf) $b(t)=\mu e^{-\mu t}, t \geq 0$, where the parameter $\mu$ is a real, positive constant. The Laplace transform (LT) is $\bar{b}(s)=\mu /(\mu+s)$. The mean, second moment, and variance of the exponential distribution are $\nu_{1}=1 / \mu, \quad \nu_{2}=2 / \mu^{2}$, and $\sigma^{2}=1 / \mu^{2}$, respectively. Substituting the LT of exponential service-time distribution $\bar{b}(s)$ in functional equation (2), we have

$$
R(z)=z \bar{b}(\lambda-\lambda R(z))=\frac{\mu z}{\mu+\lambda-\lambda R(z)} .
$$

Using (16) in (5) and (6), we get

$$
\begin{aligned}
P\left(N_{m}=n\right)= & \frac{m}{n(n-m) !} D_{z}^{n-m}\left(\frac{\mu}{\mu+\lambda-\lambda z}\right)_{z=0}^{n} \\
= & \frac{m}{n(n-m) !} \mu^{n}\left\{D_{z}^{n-m}(\mu+\lambda-\lambda z)^{-n}\right\}_{z=0} \\
= & \frac{m}{n(n-m) !} \mu^{n} \lambda^{n-m} n(n+1) \ldots(2 n-m-1) \\
& \cdot\left\{(\mu+\lambda-\lambda z)^{-2 n+m}\right\}_{z=0} \\
= & \frac{m}{n}\left(\begin{array}{c}
2 n-m-1 \\
n-1
\end{array}\right) \frac{\rho^{n-m}}{(1+\rho)^{2 n-m}} \\
n= & m, m+1, \ldots,
\end{aligned}
$$

which matches with the results of Medhi [20]. From (14) and (15), the mean and variance of the number of customers served during a busy period when initiated by $m$ customers can be obtained as follows:

$$
\begin{aligned}
E\left(N_{m}\right) & =\frac{m}{1-\rho} \\
\operatorname{Var}\left(N_{m}\right) & =\frac{m \rho(1+\rho)}{(1-\rho)^{3}} .
\end{aligned}
$$

When $m=1$, the distribution of the number of customers served during a busy period initiated by a single customer is

$$
P(N=n)=\frac{1}{n}\left(\begin{array}{c}
2 n-2 \\
n-1
\end{array}\right) \frac{\rho^{n-1}}{(1+\rho)^{2 n-1}}
$$

Remark. We could have obtained mean and variance from the distribution presented above in equation (17), but we have used (14) and (15) to find the mean and variance, respectively. This is because, in simple cases like the one presented in equation (17), we can find the mean and variance easily, but in more complex situations that arise later, it is not that simple to get the mean and variance from the distributions derived there. However, the accuracy of the analytic results was checked numerically.

3.2. Deterministic Distribution. We consider the deterministic distribution with pdf $b(t)=\delta(t-k)$, where $\delta(\cdot)$ is the Dirac delta function. The LT is given by $\bar{b}(s)=e^{-s \cdot k}$. The mean, second moment, and variance of the deterministic distribution are $\nu_{1}=k, v_{2}=k^{2}$, and $\sigma^{2}=0$, respectively. Substituting the LT of deterministic service-time distribution in functional equation (2), we have 


$$
R(z)=z\left(e^{-(\lambda-\lambda R(z)) k}\right)
$$

Using (20) in (5) and (6), we get

$$
\begin{aligned}
P\left(N_{m}=n\right) & =\frac{m}{n(n-m) !}\left\{D_{z}^{n-m}\left(e^{-(\lambda-\lambda z) k}\right)^{n}\right\}_{z=0} \\
& =\frac{m}{n(n-m) !} e^{-\lambda n k}\left\{D_{z}^{n-m}\left(e^{\lambda n k z}\right)\right\}_{z=0} \\
& =\frac{m}{n(n-m) !} e^{-\lambda n k}(\lambda n k)^{n-m} \\
& =\frac{m}{(n-m) !} n^{n-m-1} e^{-n \rho}(\rho)^{n-m} \\
& \quad n=m, m+1, \ldots,
\end{aligned}
$$

which is the Borel-Tanner distribution with $\rho=\lambda k$. This corresponds to the distribution derived by Tanner [5] as the distribution of the number of customers served during a busy period. The mean and variance of the distribution of the number of customers served during a busy period can be obtained as

$$
\begin{aligned}
E\left(N_{m}\right) & =\frac{m}{1-\rho}, \\
\operatorname{Var}\left(N_{m}\right) & =\frac{m \rho}{(1-\rho)^{3}},
\end{aligned}
$$

which match with the results reported in [20].

3.3. Erlang- $k$ Distribution. A random variable $X$ has an Erlang- $k(k=1,2, \ldots)$ distribution if $X$ is the sum of $k$ independent random variables $X_{1}, \ldots, X_{k}$ having a common exponential distribution with mean $1 / \mu$. The probability density function of the Erlang- $k$ distribution is $b(t)=\mu(\mu t)^{k-1} e^{-\mu t} /(k-1) !, \mu>0$ and $t \geq 0, k=1,2, \ldots$ The Laplace transform is given by $\bar{b}(s)=(\mu /(\mu+s))^{k}$. The Erlang distribution is a special case of the gamma distribution. It is defined by two parameters, $\mu$ and $k$, where $\mu$ is the scale parameter and $k$ is the shape parameter which must be a positive integer. In the gamma distribution, $k$ can be any real number, including fractions. The mean, second moment, and variance of the Erlang- $k$ distribution are $\nu_{1}=k / \mu$, $v_{2}=\left(k / \mu^{2}\right)(1+k)$, and $\sigma^{2}=k / \mu^{2}$, respectively. Substituting the LT of Erlang- $k$ service-time distribution in functional equation (2), we have

$$
R(z)=z\left(\frac{\mu}{\mu+\lambda-\lambda R(z)}\right)^{k}
$$

Using (23) in (5) and (6), we get

$$
\begin{aligned}
P\left(N_{m}=n\right)= & \frac{m}{n(n-m) !}\left\{D_{z}^{n-m}\left(\frac{\mu}{\mu+\lambda-\lambda z}\right)^{n k}\right\}_{z=0} \\
= & \frac{m}{n(n-m) !} \mu^{n k}\left\{D_{z}^{n-m}(\mu+\lambda-\lambda z)^{-n k}\right\}_{z=0} \\
= & \frac{m}{n} \frac{n k(n k+1) \ldots(n k+n-m-1)}{(n-m) !} \\
& \cdot\left(\frac{\lambda}{\mu}\right)^{n-m}\left(\frac{\mu}{\mu+\lambda}\right)^{n k+n-m}, \quad n=m, m+1, \ldots \\
= & \frac{m}{n}\left(\begin{array}{c}
n k+n-m-1 \\
n-m
\end{array}\right)\left(\frac{\lambda}{\mu}\right)^{n-m} \\
& \cdot\left(\frac{\mu}{\mu+\lambda}\right)^{n k+n-m} \quad, \quad n=m, m+1, \ldots
\end{aligned}
$$

The mean and variance of the number of customers served during a busy period can be obtained as

$$
\begin{aligned}
E\left(N_{m}\right) & =\frac{m}{1-\rho}, \\
\operatorname{Var}\left(N_{m}\right) & =\frac{m \rho(k+\rho)}{k(1-\rho)^{3}},
\end{aligned}
$$

where $\rho=\lambda k / \mu$.

3.4. Gamma Distribution. Let us consider the gamma distribution with probability density function $b(t)=\mu(\mu t)^{\gamma-1} e^{-\mu t} / \Gamma(\gamma), \quad \mu>0, \gamma>0, t \geq 0, \quad$ and $\quad \Gamma(\gamma)=$ $\int_{0}^{\infty} t^{\gamma-1} e^{-t} d t$ for $\gamma>0$. The parameters are $\mu>0$ and $\gamma>0$. The parameters $\gamma$ and $\mu$ are called the shape and scale parameters, respectively. The mean, second moment, and variance of the gamma distribution are $\nu_{1}=\gamma / \mu$, $v_{2}=\left(\gamma / \mu^{2}\right)(1+\gamma)$, and $\sigma^{2}=\left(\gamma / \mu^{2}\right)$, respectively. The Laplace transform is $\bar{b}(s)=(\mu /(\mu+s))^{\gamma}$. It resembles the function involved in defining the Erlang- $k$ distribution. Substituting the LT of gamma service-time distribution in functional equation (2) and using Lagrange's inversion theorem, the distribution of the number of customers served during a busy period initiated by $m$ customers is

$$
\begin{aligned}
P\left(N_{m}=n\right) & =\frac{m}{n(n-m) !}\left\{D_{z}^{n-m}\left(\frac{\mu z}{\mu+\lambda-\lambda z}\right)^{\gamma n}\right\}_{z=0} \\
& =\frac{m}{n}\left(\begin{array}{c}
n \gamma+n-m-1 \\
n-m
\end{array}\right)\left(\frac{\mu}{\mu+\lambda}\right)^{\gamma n}\left(\frac{\lambda}{\mu+\lambda}\right)^{n-m} .
\end{aligned}
$$


The mean and variance of the number of customers served during a busy period can be obtained as

$$
\begin{aligned}
E\left(N_{m}\right) & =\frac{m}{1-\rho}, \\
\operatorname{Var}\left(N_{m}\right) & =\frac{m \rho(\gamma+\rho)}{\gamma(1-\rho)^{3}} .
\end{aligned}
$$

3.5. Chi-Square Distribution. Let $Y_{1}, Y_{2}, \ldots, Y_{k}$ be normal independent random variables with mean 0 and variance 1 . Then, the random variable $\chi^{2}=Y_{1}^{2}+Y_{2}^{2}+\ldots Y_{k}^{2}$ has the probability density function $b(t)=\left(t^{k / 2-1} e^{-t / 2} /\right.$ $\left.2^{k / 2} \Gamma(k / 2)\right), t>0$, and is said to follow the chi-square distribution with $k$ degrees of freedom, abbreviated $\chi_{k}^{2}$. The mean, second moment, and variance of the $\chi_{k}^{2}$ distribution are $v_{1}=k, v_{2}=k(2-k)$, and $\sigma^{2}=2 k$, respectively. The special case of the gamma distribution is the chi-square distribution by setting $\gamma=k / 2$ and $\mu=1 / 2$. The LT is $\bar{b}(s)=(1 /(1+2 s))^{k / 2}$. Substituting the LT of chi-square service-time distribution in functional equation (2) and using Lagrange's inversion theorem, the distribution of the number of customers served during a busy period initiated by $m$ customers is

$$
\begin{aligned}
P\left(N_{m}=n\right)= & \frac{m}{n(n-m) !}\left\{D_{z}^{n-m}\left(\frac{1}{(1+2(\lambda-\lambda z))^{k / 2}}\right)^{n}\right\}_{z=0} \\
= & \frac{m}{n(n-m) !} \frac{1}{(1+2 \lambda)^{n k / 2}} \\
& \cdot\left\{D_{z}^{n-m}\left(1-\frac{2 \lambda z}{1+2 \lambda}\right)^{n k / 2}\right\}_{z=0} \\
= & \frac{m}{n}\left(\frac{n k}{2}+n-m-1\right) \frac{1}{(1+2 \lambda)^{n k / 2}} \\
& \cdot\left(\frac{2 \lambda}{1+2 \lambda}\right)^{n-m} \cdot
\end{aligned}
$$

The mean and variance of the number of customers served during a busy period can be obtained as

$$
\begin{aligned}
E\left(N_{m}\right) & =\frac{m}{1-\lambda k} \\
\operatorname{Var}\left(N_{m}\right) & =\frac{m k \lambda(1+2 \lambda)}{(1-k \lambda)^{3}} .
\end{aligned}
$$

3.6. Inverse Gaussian Distribution. We consider the inverse Gaussian distribution with pdf $b(t)=\sqrt{\alpha /\left(2 \pi t^{3}\right)}$ $e^{-\alpha(t-\beta)^{2} /\left(2 \beta^{2} t\right)}$ and LT $\bar{b}(s)=e^{\phi(1-\sqrt{1+(2 s / \gamma)})}$, where $\phi=\alpha / \beta$ and $\gamma=\beta^{2} / \alpha$. The mean, second moment, and variance of the inverse Gaussian distribution are $\nu_{1}=\phi / \gamma$, $v_{2}=\phi(1+\phi) / \gamma^{2}$, and $\sigma^{2}=\phi / \gamma^{2}$, respectively. Substituting the LT of inverse Gaussian service-time distribution in functional equation (2) and applying Lagrange's inversion theorem, we get

$$
\begin{aligned}
& \left.P\left(N_{m}=n\right)=\frac{m}{n(n-m) !} D_{z}^{n-m}\left(e^{\phi\left(1-(1+(2(\lambda-\lambda z) / \gamma))^{1 / 2}\right.}\right)\right)_{z=0}^{n} \\
& =\frac{m}{n(n-m) !} D_{z}^{n-m}\left(e^{n \phi} e^{-n \phi(1+(2(\lambda-\lambda z) / \gamma))^{1 / 2}}\right)_{z=0} \\
& =\frac{m}{n(n-m) !} e^{n \phi} D_{z}^{n-m} \\
& \cdot\left(e^{-n \phi(1+(2 \lambda / \gamma))^{1 / 2}(1-(2 \lambda z /(2 \lambda+\gamma)))^{1 / 2}}\right)_{z=0} \\
& =\frac{m}{n(n-m) !} e^{n \phi} \sum_{i=0}^{\infty} \frac{(-n \phi)^{i}(1+(2 \lambda / \gamma))^{i / 2}}{i !} \\
& \cdot D_{z}^{n-m}\left[\left(1-\frac{2 \lambda z}{2 \lambda+\gamma}\right)^{i / 2}\right]_{z=0} \\
& =\frac{m}{n} e^{n \phi} \sum_{i=0}^{\infty}(-1)^{n-m+i} \frac{(n \phi)^{i}(1+(2 \lambda / \gamma))^{i / 2}}{i !} \\
& \cdot\left(\begin{array}{c}
\frac{i}{2} \\
n-m
\end{array}\right)\left(\frac{2 \lambda}{2 \lambda+\gamma}\right)^{n-m}
\end{aligned}
$$

The mean and variance of the number of customers served during a busy period can be obtained as

$$
\begin{aligned}
E\left(N_{m}\right) & =\frac{m \gamma}{\gamma-\lambda \phi}, \\
\operatorname{Var}\left(N_{m}\right) & =\frac{m \lambda \phi \gamma(\lambda+\gamma)}{(\gamma-\lambda \phi)^{3}} .
\end{aligned}
$$

3.7. Generalized Erlang Distribution. We assume the generalized Erlang distribution or hypoexponential distribution of a series of 2 exponential distributions each with their own rates $\mu_{1}$ and $\mu_{2}$ with probability density function $b(t)=$ $\left(\mu_{1} \mu_{2} /\left(\mu_{1}-\mu_{2}\right)\right)\left(e^{-\mu_{2} t}-e^{-\mu_{1} t}\right), \mu_{1}, \mu_{2}>0, t>0$, and Laplace transform $\bar{b}(s)=\left(\mu_{1} \mu_{2} /\left(s+\mu_{1}\right)\left(s+\mu_{2}\right)\right)$. The mean, second moment, and variance of the Generalized Erlang 
distribution are $\nu_{1}=\left(1 / \mu_{1}\right)+\left(1 / \mu_{2}\right), \quad v_{2}=\left(2 / \mu_{1}^{2}\right)+(2 /$ $\left.\mu_{1} \mu_{2}\right)+\left(2 / \mu_{2}^{2}\right)$, and $\sigma^{2}=\left(1 / \mu_{1}^{2}\right)+\left(1 / \mu_{2}^{2}\right)$, respectively.

Substituting the LT of generalized Erlang service-time distribution $\bar{b}(s)$ in functional equation (2) and applying Lagrange's inversion theorem, we get

$$
\begin{aligned}
& P\left(N_{m}=n\right)=\frac{m}{n(n-m) !} \prod_{i=1}^{2}\left(\frac{\mu_{i}}{\mu_{i}+\lambda}\right)^{n} \\
& \cdot D_{z}^{n-m}\left\{\left(1-\frac{\lambda z}{\mu_{1}+\lambda}\right)^{-n}\left(1-\frac{\lambda z}{\mu_{2}+\lambda}\right)^{-n}\right\}_{z=0} \\
& =\frac{m}{n(n-m) !} \prod_{i=1}^{2}\left(\frac{\mu_{i}}{\mu_{i}+\lambda}\right)^{n} \sum_{k=0}^{n-m}\left(\begin{array}{c}
n-m \\
k
\end{array}\right) \\
& \cdot\left\{D_{z}^{n-m-k}\left(1-\frac{\lambda z}{\mu_{1}+\lambda}\right)^{-n}\right\}_{z=0} \\
& \times\left\{D_{z}^{k}\left(1-\frac{\lambda z}{\mu_{2}+\lambda}\right)^{-n}\right\}_{z=0} \\
& =\frac{m}{n} \prod_{i=1}^{2}\left(\frac{\mu_{i}}{\mu_{i}+\lambda}\right)^{n} \sum_{k=0}^{n-m}\left(\begin{array}{c}
n+k-1 \\
k
\end{array}\right) \\
& \cdot\left(\begin{array}{c}
2 n-m-k-1 \\
n-m-k
\end{array}\right)\left(\frac{\lambda}{\mu_{1}+\lambda}\right)^{n-m-k} \\
& \times\left(\frac{\lambda}{\mu_{2}+\lambda}\right)^{k}
\end{aligned}
$$

The mean and variance of the number of customers served during a busy period can be obtained as

$$
\begin{aligned}
E\left(N_{m}\right) & =\frac{m \mu_{1} \mu_{2}}{\mu_{1} \mu_{2}-\lambda\left(\mu_{1}+\mu_{2}\right)}, \\
\operatorname{Var}\left(N_{m}\right) & =\frac{m \lambda \mu_{1} \mu_{2}\left[\mu_{1} \mu_{2}\left(\mu_{1}+\mu_{2}\right)+\lambda\left(\mu_{1}^{2}+\mu_{2}^{2}\right)\right]}{\left[\mu_{1} \mu_{2}-\lambda\left(\mu_{1}+\mu_{2}\right)\right]^{3}} .
\end{aligned}
$$

3.8. Matrix Exponential Distribution. We consider the matrix exponential distribution with pdf $b(t)=$ $\left(1+\left(1 / 4 \pi^{2}\right)\right)(1-\cos (2 \pi t)) e^{-t}$ and Laplace transform $\bar{b}(s)=\left((a+1) /\left((s+1)(s+1)^{2}+a\right)\right)$, where $a=4 \pi^{2}$. The mean, second moment, and variance of the matrix exponential distribution are $v_{1}=((a+3) /(a+1)), \quad v_{2}=$ $\left(2\left(a^{2}+3 a+6\right) /(a+1)^{2}\right)$, and $\sigma^{2}=\left(a^{2}+3\right) /(a+1)^{2}$, respectively. Substituting the LT of matrix exponential servicetime distribution $\bar{b}(s)$ in functional equation (2) and using Lagrange's expansion, we have

$$
\begin{aligned}
& P\left(N_{m}=n\right)=\frac{m}{n(n-m) !} \\
& \cdot\left\{D_{z}^{n-m}\left(\frac{a+1}{(\lambda-\lambda z+1)\left((\lambda-\lambda z+1)^{2}+a\right)}\right)^{n}\right\}_{z=0} \\
& =\frac{m}{n(n-m) !}(a+1)^{n}(1+\lambda)^{-n}\left((1+\lambda)^{2}+a\right)^{-n} \\
& \times D_{z}^{n-m}\left\{\left(1-\frac{\lambda z}{1+\lambda+\sqrt{-a}}\right)^{-n}\right. \\
& \left.\cdot\left(1-\frac{\lambda z}{1+\lambda-\sqrt{-a}}\right)^{-n}\left(1-\frac{\lambda z}{1+\lambda}\right)^{-n}\right\}_{z=0} \\
& =\frac{m}{n(n-m) !}(a+1)^{n}(1+\lambda)^{-n}\left((1+\lambda)^{2}+a\right)^{-n} \\
& \cdot \sum_{i=0}^{n-m} \sum_{j=0}^{n-m-i} \frac{(n-m) !}{i ! j !(n-m-i-j) !} \\
& \times\left\{D_{z}^{i}\left(1-\frac{\lambda z}{1+\lambda+\sqrt{-a}}\right)^{-n}\right\}_{z=0} \\
& \left\{D_{z}^{j}\left(1-\frac{\lambda z}{1+\lambda-\sqrt{-a}}\right)^{-n}\right\}_{z=0} \\
& \times\left\{D_{z}^{n-m-i-j}\left(1-\frac{\lambda z}{1+\lambda}\right)^{-n}\right\}_{z=0} \\
& =\frac{m}{n}\left(\frac{a+1}{1+\lambda}\right)^{n}\left((1+\lambda)^{2}+a\right)^{-n} \sum_{i=0}^{n-m} \sum_{j=0}^{n-m-i} \\
& \cdot\left(\begin{array}{c}
n+i-1 \\
i
\end{array}\right)\left(\begin{array}{c}
n+j-1 \\
j
\end{array}\right) \\
& \times\left(\begin{array}{c}
2 n-m-i-j-1 \\
n-m-i-j
\end{array}\right)\left(\frac{\lambda}{1+\lambda+\sqrt{-a}}\right)^{i} \\
& \cdot\left(\frac{\lambda}{1+\lambda-\sqrt{-a}}\right)^{j}\left(\frac{\lambda}{1+\lambda}\right)^{n-m-i-j} .
\end{aligned}
$$

The mean and variance of the number of customers served during a busy period is

$$
\begin{aligned}
E\left(N_{m}\right) & =\frac{m(a+1)}{a+1-\lambda(a+3)} \\
\operatorname{Var}\left(N_{m}\right) & =\frac{m(a+1)\left[\lambda(a+1)(a+3)+\lambda^{2}\left(a^{2}+3\right)\right]}{[a+1-\lambda(a+3)]^{3}} .
\end{aligned}
$$


3.9. Hyperexponential Distribution. We assume the hyperexponential distribution with probability density function $b(t)=\sum_{i=1}^{k} p_{i} \mu_{i} e^{-\mu_{i} t}, t>0$, such that $\sum_{i=0}^{k} p_{i}=1$ and Laplace transform $\vec{b}(s)=\sum_{i=1}^{k}\left(p_{i} \mu_{i} /\left(\mu_{i}+s\right)\right)$. The mean, second moment, and variance of the hyperexponential distribution are $\nu_{1}=\sum_{i=1}^{k} p_{i} / \mu_{i}, \nu_{2}=\sum_{i=1}^{k} 2 p_{i} / \mu_{i}^{2}$, and $\sigma^{2}=\sum_{i=1}^{k} p_{i} / \mu_{i}$, respectively. Taking $k=2$ and substituting the LT of hyperexponential service-time distribution $\bar{b}(s)=\sum_{i=1}^{2} p_{i} \mu_{i} /$ $\left(\mu_{i}+s\right)$ in functional equation (2), we have

$$
R(z)=z\left(\frac{p_{1} \mu_{1}}{\mu_{1}+\lambda-\lambda R(z)}+\frac{p_{2} \mu_{2}}{\mu_{2}+\lambda-\lambda R(z)}\right) .
$$

Applying Lagrange's expansion and Leibniz rule, we get

$$
\begin{aligned}
& P\left(N_{m}=n\right)=\frac{m}{n(n-m) !}\left\{D_{z}^{n-m}\left(\frac{p_{1} \mu_{1}}{\mu_{1}+\lambda-\lambda z}+\frac{p_{2} \mu_{2}}{\mu_{2}+\lambda-\lambda z}\right)^{n}\right\}_{z=0} \\
& =\frac{m}{n(n-m) !} D_{z}^{n-m}\left(\frac{p_{1} \mu_{1}\left(\mu_{2}+\lambda-\lambda z\right)+p_{2} \mu_{2}\left(\mu_{1}+\lambda-\lambda z\right)}{\left(\mu_{1}+\lambda-\lambda z\right)\left(\mu_{2}+\lambda-\lambda z\right)}\right)_{z=0}^{n} \\
& =\frac{m}{n(n-m) !} \prod_{i=1}^{2}\left(\mu_{i}+\lambda\right)^{-n} \sum_{i=0}^{n-m} \sum_{j=0}^{n-m-i} \frac{(n-m) !}{i ! j !(n-m-i-j) !} D_{z}^{n-m-i-j}\left(1-\frac{\lambda z}{\mu_{1}+\lambda}\right)_{z=0}^{-n} \\
& \cdot D_{z}^{i}\left(1-\frac{\lambda z}{\mu_{2}+\lambda}\right)_{z=0}^{-n} D_{z}^{j}\left[p_{1} \mu_{1}\left(\mu_{2}+\lambda\right)\left(1-\frac{\lambda z}{\mu_{2}+\lambda}\right)+p_{2} \mu_{2}\left(\mu_{1}+\lambda\right)\left(1-\frac{\lambda z}{\mu_{1}+\lambda}\right)\right]_{z=0}^{n} \\
& =\frac{m}{n} \prod_{i=1}^{2}\left(\mu_{i}+\lambda\right)^{-n} \sum_{i=0}^{n-m} \sum_{j=0}^{n-m-i}\left(\begin{array}{c}
2 n-m-i-j-1 \\
n-m-i-j
\end{array}\right)\left(\begin{array}{c}
n+i-1 \\
i
\end{array}\right)\left(\frac{\lambda}{\mu_{1}+\lambda}\right)^{n-m-i-j}\left(\frac{\lambda}{\mu_{2}+\lambda}\right)^{i} \\
& \cdot \sum_{k=0}^{n}\left(\begin{array}{l}
n \\
k
\end{array}\right)\left(p_{1} \mu_{1}\left(\mu_{2}+\lambda\right)\right)^{n-k}(-1)^{j}\left(p_{2} \mu_{2}\left(\mu_{1}+\lambda\right)\right)^{k} \sum_{l=0}^{j}\left(\begin{array}{c}
k \\
j-l
\end{array}\right)\left(\frac{\lambda}{\mu_{1}+\lambda}\right)^{j-l}\left(\begin{array}{c}
n-k \\
l
\end{array}\right)\left(\frac{\lambda}{\mu_{2}+\lambda}\right)^{l} .
\end{aligned}
$$

The mean and variance of the number of customers served during a busy period can be obtained from as

$$
\begin{aligned}
E\left(N_{m}\right) & =\frac{m \mu_{1} \mu_{2}}{\mu_{1} \mu_{2}-\lambda\left(p_{1} \mu_{2}+p_{2} \mu_{1}\right)}, \\
\operatorname{Var}\left(N_{m}\right) & =\frac{m \lambda \mu_{1} \mu_{2}\left[\left(p_{1} \mu_{2}+p_{2} \mu_{1}\right)\left(\mu_{1} \mu_{2}-\lambda p_{1} \mu_{2}-\lambda p_{2} \mu_{1}\right)+2 \lambda\left(p_{1} \mu_{2}^{2}+p_{2} \mu_{1}^{2}\right)\right]}{\left(\mu_{1} \mu_{2}-\lambda p_{1} \mu_{2}-\lambda p_{2} \mu_{1}\right)^{3}} .
\end{aligned}
$$

3.10. Uniform Distribution. We assume the uniform distribution with probability density function $b(t)=$ $1 /(b-a), a \leq t \leq b$, where $a$ and $b$ are real constants with $a<b$. The Laplace transform is $\bar{b}(s)=\left(e^{-a s}-e^{-b s}\right) /((b-a) s)$. The mean, second moment, and variance of the uniform distribution are $v_{1}=(a+b) / 2, \quad v_{2}=\left(b^{2}+a b+a^{2}\right) / 3$, and $\sigma^{2}=(b-a)^{2} / 12$, respectively. Substituting the LT of uniform service-time distribution $\bar{b}(s)$ in functional equation (2) and applying Lagrange's inversion theorem, we get 


$$
\begin{aligned}
P\left(N_{m}=n\right) & =\frac{m}{n(n-m) !} D_{z}^{n-m}\left(\frac{e^{-a(\lambda-\lambda z)}-e^{-b(\lambda-\lambda z)}}{(b-a)(\lambda-\lambda z)}\right)_{z=0}^{n} \\
& =\frac{m}{n(n-m) !}(b-a)^{-n} \lambda^{-n} D_{z}^{n-m}\left[\left(e^{-a(\lambda-\lambda z)}-e^{-b(\lambda-\lambda z)}\right)^{n}(1-z)^{-n}\right]_{z=0} \\
& =\frac{m}{n(n-m) !}(b-a)^{-n} \lambda^{-n} e^{-a \lambda n} D_{z}^{n-m}\left[e^{a \lambda n z}\left(1-e^{-(b-a) \lambda(1-z)}\right)^{n}(1-z)^{-n}\right]_{z=0} \\
& =\frac{m}{n(n-m) !}(b-a)^{-n} \lambda^{-n} e^{-a \lambda n} \sum_{i=0}^{n-m} \sum_{j=0}^{n-m-i} \frac{(n-m) !}{i ! j !(n-m-i-j) !} \times D_{z}^{i}\left(e^{a \lambda n z}\right)_{z=0} D_{z}^{j}\left(1-e^{-(b-a) \lambda(1-z)}\right)_{z=0}^{n} D_{z}^{n-m-i-j}(1-z)_{z=0}^{-n} \\
& =\frac{m(b-a)^{-n} \lambda^{-n} e^{-a \lambda n}}{n} \sum_{i=0}^{n-m} \frac{(a \lambda n)^{i}}{i !} \sum_{j=0}^{n-m-i}\left(\begin{array}{c}
2 n-m-i-j-1 \\
n-m-i-j
\end{array}\right) \times \sum_{k=0}^{n}\left(\begin{array}{l}
n \\
k
\end{array}\right)(-1)^{k} e^{-(b-a) \lambda k} \frac{((b-a) \lambda k)^{j}}{j !} .
\end{aligned}
$$

The mean and variance of the number of customers served during a busy period can be obtained as

$$
\begin{aligned}
E\left(N_{m}\right) & =\frac{2 m}{2-\lambda(a+b)}, \\
\operatorname{Var}\left(N_{m}\right) & =\frac{2 m \lambda\left(6(a+b)+\lambda(b-a)^{2}\right)}{3(2-\lambda(a+b))^{3}} .
\end{aligned}
$$

3.11. Two-Phase Coxian Distribution: $C o x-C_{2}$. The Coxian distribution is a generalization of the hypoexponential distribution. Instead of only being able to get into the absorbing state from state $k$, it can be arrived from any phase. Here, we assume that $b(t)$ follows the two-phase Coxian distribution and its Laplace transform is $\bar{b}(s)=(1-\alpha) \mu_{1} /$ $\left(\mu_{1}+s\right)+\left(\alpha \mu_{1} \mu_{2} /\left(\mu_{1}+s\right)\left(\mu_{2}+s\right)\right)$. The mean, second moment, and variance of the $C_{2}$ distribution are $\nu_{1}=$ $\left(1 / \mu_{1}\right)+\left(\alpha / \mu_{2}\right), \quad v_{2}=\left(2 / \mu_{1}^{2}\right)+\left(2 \alpha / \mu_{2}^{2}\right)+\left(2 \alpha / \mu_{1} \mu_{2}\right), \quad$ and $\sigma^{2}=\left(\left(\mu_{2}^{2}+\alpha \mu_{1}^{2}(2-\alpha)\right) / \mu_{1}^{2} \mu_{2}^{2}\right)$, respectively.

Substituting the LT of Cox $C_{2}$ service-time distribution $\bar{b}(s)$ in functional equation (2) and using Lagrange's inversion theorem and Leibniz's rule, we have

$$
\begin{aligned}
& P\left(N_{m}=n\right)=\frac{m}{n(n-m) !}\left\{D_{z}^{n-m}\left(\frac{(1-\alpha) \mu_{1}}{\mu_{1}+\lambda-\lambda z}+\frac{\alpha \mu_{1} \mu_{2}}{\left(\mu_{1}+\lambda-\lambda z\right)\left(\mu_{2}+\lambda-\lambda z\right)}\right)^{n}\right\}_{z=0} \\
& =\frac{m}{n(n-m) !} D_{z}^{n-m}\left(\frac{(1-\alpha) \mu_{1}\left(\mu_{2}+\lambda-\lambda z\right)+\alpha \mu_{1} \mu_{2}}{\left(\mu_{1}+\lambda-\lambda z\right)\left(\mu_{2}+\lambda-\lambda z\right)}\right)_{z=0}^{n} \\
& =\frac{m}{n(n-m) !} \prod_{i=1}^{2}\left(\mu_{i}+\lambda\right)^{-n} D_{z}^{n-m}\left\{\left(1-\frac{\lambda z}{\mu_{1}+\lambda}\right)^{-n}\left(1-\frac{\lambda z}{\mu_{2}+\lambda}\right)^{-n} \times\left(\mu_{1} \mu_{2}+(1-\alpha) \mu_{1} \lambda-(1-\alpha) \mu_{1} \lambda z\right)^{n}\right\}_{z=0} \\
& =\frac{m}{n(n-m) !} \prod_{i=1}^{2}\left(\mu_{i}+\lambda\right)^{-n} \mu_{1}^{n}\left(\mu_{2}+(1-\alpha) \lambda\right)^{n} \sum_{i=0}^{n-m} \sum_{j=0}^{n-m-i} \frac{(n-m) !}{i ! j !(n-m-i-j) !} \times D_{z}^{n-m-i-j}\left(1-\frac{\lambda z}{\mu_{1}+\lambda}\right)_{z=0}^{-n} \\
& \cdot D_{z}^{i}\left(1-\frac{\lambda z}{\mu_{2}+\lambda}\right)_{z=0}^{-n} D_{z}^{j}\left(1-\frac{(1-\alpha) \lambda z}{\mu_{2}+(1-\alpha) \lambda}\right)_{z=0}^{n} \\
& =\frac{m}{n} \prod_{i=1}^{2}\left(\mu_{i}+\lambda\right)^{-n} \mu_{1}^{n}\left(\mu_{2}+(1-\alpha) \lambda\right)^{n} \sum_{i=0}^{n-m} \sum_{j=0}^{n-m-i}\left(\begin{array}{c}
2 n-m-i-j-1 \\
n-m-i-j
\end{array}\right)\left(\begin{array}{c}
n+i-1 \\
i
\end{array}\right) \\
& \times\left(\begin{array}{c}
n \\
j
\end{array}\right)(-1)^{j}\left(\frac{\lambda}{\mu_{1}+\lambda}\right)^{n-m-i-j}\left(\frac{\lambda}{\mu_{2}+\lambda}\right)^{i}\left(\frac{(1-\alpha) \lambda}{\mu_{2}+(1-\alpha) \lambda}\right)^{j} .
\end{aligned}
$$


The mean and variance of the number of customers served during a busy period can be given as

$$
\begin{aligned}
E\left(N_{m}\right) & =\frac{m \mu_{1} \mu_{2}}{\mu_{1} \mu_{2}-\lambda\left(\mu_{2}+\alpha \mu_{1}\right)}, \\
\operatorname{Var}\left(N_{m}\right) & =\frac{m \lambda \mu_{1} \mu_{2}\left[\mu_{2}^{2}\left(\lambda+\mu_{1}\right)+\alpha \mu_{1}^{2}\left(\mu_{2}+2 \lambda-\alpha \lambda\right)\right]}{\left[\mu_{1} \mu_{2}-\lambda\left(\mu_{2}+\alpha \mu_{1}\right)\right]^{3}} .
\end{aligned}
$$

3.12. Phase-Type Distribution. A phase-type (PH) distribution is constructed by a mixture of exponentially distributed phases. It results from a system of one or more inter-related Poisson processes occurring in sequence, or phases. Let $\omega$ denote the number of phases. The process begins in phase $i$ with probability $\alpha_{i}, i=1,2, \ldots, \omega$. Let $\mu_{i}, i=1,2, \ldots, \omega$ be the transition rate out of phase $i$. After completion of a phase, the process may join the absorbing state or continue with a nonabsorbing phase. Let $r_{i \omega}$ be the probability of transition from phase $i$ to phase $j, i, j=1,2, \ldots, \omega$, and let $r_{i 0}$ be the probability of transition from phase $i$ to the absorbing state, $i=1,2, \ldots, \omega$. The parameters must satisfy the following conditions: $\sum_{i=1}^{\omega} \alpha_{i}=1$ and $r_{i 0}=1-\sum_{j=1}^{\omega} r_{i j}, i=1,2$, $\ldots, \omega$. Let the row vector $\alpha$ denote the $\omega$-dimensional vector of the initial state distribution of the underlying Markov process, the square matrix $T$ denote the sub-generator of the underlying Markov process held to the transient states $1, \ldots, \omega$. Let $\mathbf{r}=\mathbf{T} 1$ denote the $\omega$-dimensional column vector of transition rates to the absorbing state. Hence,

$$
\begin{aligned}
\alpha & =\left(\begin{array}{cccc}
\alpha_{1} & \alpha_{2} & \cdots & \alpha_{\omega}
\end{array}\right), \\
\mathbf{T} & =\left(\begin{array}{cccc}
-\mu_{1} & \mu_{1} \mathbf{r}_{12} & \cdots & \mu_{1} \mathbf{r}_{1 \omega} \\
\mu_{2} \mathbf{r}_{21} & -\mu_{2} & \cdots & \mu_{2} \mathbf{r}_{2 \omega} \\
\vdots & \vdots & \ddots & \vdots \\
\mu_{\omega} \mathbf{r}_{\omega 1} & \mu_{\omega} \mathbf{r}_{\omega 2} & \cdots & -\mu_{\omega}
\end{array}\right), \\
\mathbf{r} & =\left(\begin{array}{c}
-\mu_{1} \mathbf{r}_{10} \\
-\mu_{2} \mathbf{r}_{20} \\
\vdots \\
-\mu_{\omega} \mathbf{r}_{\omega 0}
\end{array}\right) .
\end{aligned}
$$

The LT of phase-type distribution for the case $\omega=2$, that is, $\mathrm{PH}_{2}$ is given by

$$
\bar{b}(s)=\frac{\left(\alpha_{1} r_{10} \mu_{1}+\alpha_{2} r_{20} \mu_{2}\right) s+\left(1-r_{12} r_{21}\right) \mu_{1} \mu_{2}}{\left(s+\mu_{1}\right)\left(s+\mu_{2}\right)-r_{12} r_{21} \mu_{1} \mu_{2}}, \quad s \geq 0 .
$$

Substituting the LT of $\mathrm{PH}_{2}$ service-time distribution $\bar{b}(s)$ in functional equation (2) and using Lagrange's inversion theorem, we get

$$
\begin{aligned}
P\left(N_{m}=n\right) & =\frac{m}{n(n-m) !} D_{z}^{n-m}\left(\frac{\left(\alpha_{1} r_{10} \mu_{1}+\alpha_{2} r_{20} \mu_{2}\right)(\lambda-\lambda z)+\left(1-r_{12} r_{21}\right) \mu_{1} \mu_{2}}{\left(\lambda-\lambda z+\mu_{1}\right)\left(\lambda-\lambda z+\mu_{2}\right)-r_{12} r_{21} \mu_{1} \mu_{2}}\right)_{z=0}^{n} \\
& =\frac{m}{n(n-m) !} D_{z}^{n-m}\left(\frac{\left(\alpha_{1} r_{10} \mu_{1}+\alpha_{2} r_{20} \mu_{2}\right)(\lambda-\lambda z)+\left(1-r_{12} r_{21}\right) \mu_{1} \mu_{2}}{\lambda^{2}\left(\psi_{1}-z\right)\left(\psi_{2}-z\right)}\right)_{z=0}^{n} \\
& =\frac{m}{n(n-m) !} K_{1}^{n}\left(\lambda^{2} \psi_{1} \psi_{2}\right)^{-n} D_{z}^{n-m}\left\{\left(1-\frac{z}{\psi_{1}}\right)^{-n}\left(1-\frac{z}{\psi_{2}}\right)^{-n}\left(1-K_{2} z\right)^{n}\right\}_{z=0} \\
& =\frac{m}{n} K_{1}^{n}\left(\lambda^{2} \psi_{1} \psi_{2}\right)^{-n} \sum_{i=0}^{n-m} \sum_{j=0}^{n-m-i}\left(\begin{array}{c}
2 n-m-i-j-1 \\
n-m-i-j
\end{array}\right)\left(\begin{array}{c}
n+i-1 \\
i
\end{array}\right) \times\left(\begin{array}{c}
n \\
j
\end{array}\right)(-1)^{j} \psi_{1}^{m+i+j-n} \psi_{2}^{-i} K_{2}^{j},
\end{aligned}
$$

where

$$
\begin{aligned}
\psi_{1,2} & =\frac{\mu_{1}+\mu_{2}+2 \lambda \pm \sqrt{\left(\mu_{1}-\mu_{2}\right)^{2}+4 \mu_{1} \mu_{2} r_{12} r_{21}}}{2 \lambda}, \\
K_{1} & =\lambda\left(\alpha_{1} r_{10} \mu_{1}+\alpha_{2} r_{20} \mu_{2}\right)+\mu_{1} \mu_{2}\left(1-r_{12} r_{21}\right), \\
K_{2} & =\frac{\lambda\left(\alpha_{1} r_{10} \mu_{1}+\alpha_{2} r_{20} \mu_{2}\right)}{K_{1}} .
\end{aligned}
$$

The mean and second moment of the $\mathrm{PH}_{2}$ distribution are $\nu_{1}=\left(\left(\alpha_{1}\left(\mu_{2}+\mu_{1} r_{12}\right)\right)+\left(\alpha_{2}\left(\mu_{1}+\mu_{2} r_{21}\right)\right)\right) /\left(\mu_{1} \mu_{2}\left(1-r_{12} r_{21}\right)\right)$ and $\nu_{2}=2\left[\mu_{1} \alpha_{1} r_{12}+\mu_{2} \alpha_{2} r_{21}-\mu_{1} \alpha_{1}-\mu_{2} \alpha_{2}\right] / \mu_{1} \mu_{2}\left(1-r_{12}\right.$ $\left.r_{21}\right)$, respectively. Here, $\rho=\lambda \nu_{1}$. Thus, the mean and variance of the number of customers served during a busy period is

$$
\begin{aligned}
E\left(N_{m}\right) & =\frac{m \mu_{1} \mu_{2}\left(1-r_{12} r_{21}\right)}{\mu_{1} \mu_{2}\left(1-r_{12} r_{21}\right)-\lambda\left[\alpha_{1}\left(\mu_{2}+\mu_{1} r_{12}\right)+\alpha_{2}\left(\mu_{1}+\mu_{2} r_{21}\right)\right]}, \\
\operatorname{Var}\left(N_{m}\right) & =\frac{m\left[\rho(1-\rho)+\lambda^{2} v_{2}\right]}{(1-\rho)^{3}} .
\end{aligned}
$$

3.13. Markov-Modulated Poisson Process. A Markov-modulated Poisson process (MMPP) is a doubly stochastic Poisson process whose intensities vary according to the state 
of a Markov chain. An MMPP can be categorized by the number of states the modulating Markov chain contains, for example, a Markov chain with two states and two intensities is denoted MMPP-2, or sometimes switched Poisson process (SPP). If the two intensities are equal, the model becomes an ordinary Poisson process. A two-state MMPP has only two states in its underlying modulating Markov chain, described by

$$
\begin{aligned}
& Q=\left(\begin{array}{cc}
-q_{1} & q_{1} \\
q_{2} & -q_{2}
\end{array}\right), \\
& M=\left(\begin{array}{cc}
\mu_{1} & 0 \\
0 & \mu_{2}
\end{array}\right) .
\end{aligned}
$$

The Laplace transform is given by

$$
\bar{b}(s)=\frac{s\left(q_{1} \mu_{2}^{2}+q_{2} \mu_{1}^{2}\right)+\left(q_{1} \mu_{2}+q_{2} \mu_{1}\right)\left(q_{1} \mu_{2}+\mu_{1} \mu_{2}+q_{2} \mu_{1}\right)}{\left(q_{1} \mu_{2}+q_{2} \mu_{1}\right)\left\{s^{2}+\left(q_{1}+q_{2}+\mu_{1}+\mu_{2}\right) s+\left(q_{1} \mu_{2}+\mu_{1} \mu_{2}+q_{2} \mu_{1}\right)\right\}} .
$$

Substituting the LT of MMPP-2 service-time distribution $\bar{b}(s)$ in functional equation (2) and applying Lagrange's inversion theorem, we get

$$
\begin{aligned}
P\left(N_{m}=n\right) & =\frac{m}{n(n-m) !} K_{1}^{n}\left(\lambda^{2} \psi_{1} \psi_{2}\right)^{-n} D_{z}^{n-m}\left\{\left(1-\frac{z}{\psi_{1}}\right)^{-n}\left(1-\frac{z}{\psi_{2}}\right)^{-n}\left(1-K_{2} z\right)^{n}\right\}_{z=0} \\
& =\frac{m}{n} K_{1}^{n}\left(\lambda^{2} \psi_{1} \psi_{2}\right)^{-n} \sum_{i=0}^{n-m} \sum_{j=0}^{n-m-i}\left(\begin{array}{c}
2 n-m-i-j-1 \\
n-m-i-j
\end{array}\right)\left(\begin{array}{c}
n+i-1 \\
i
\end{array}\right) \times\left(\begin{array}{c}
n \\
j
\end{array}\right)(-1)^{j} \psi_{1}^{m+i+j-n} \psi_{2}^{-i} K_{2}^{j},
\end{aligned}
$$

where

$$
\begin{aligned}
& \psi_{1,2}=\frac{\left(q_{1}+q_{2}+\mu_{1}+\mu_{2}+2 \lambda\right) \pm \sqrt{\left(q_{1}+q_{2}+\mu_{1}+\mu_{2}\right)^{2}-4\left(\mu_{1} \mu_{2}+q_{1} \mu_{2}+q_{2} \mu_{1}\right)}}{2 \lambda} \\
& K_{1}=\lambda\left(q_{1} \mu_{2}^{2}+q_{2} \mu_{1}^{2}\right)+\left(q_{1} \mu_{2}+q_{2} \mu_{1}\right)\left(q_{1} \mu_{2}+\mu_{1} \mu_{2}+q_{2} \mu_{1}\right) \\
& K_{2}=\frac{\lambda\left(q_{1} \mu_{2}^{2}+q_{2} \mu_{1}^{2}\right)}{K_{1}}
\end{aligned}
$$

The mean and second moment of the MMPP-2 distribution are $\nu_{1}=\left(q_{1}^{2} \mu_{2}+q_{2}^{2} \mu_{1}+q_{1} q_{2}\left(\mu_{1}+\mu_{2}\right)\right)+\left(\mu_{1} \mu_{2}\left(q_{1}+q_{2}\right)\right) /$ $\left(q_{1} \mu_{2}+\mu_{1} \mu_{2}+q_{2} \mu_{1}\right)$ and $\nu_{2}=2\left[\left(\left(q_{1}+q_{2}+\mu_{1}+\mu_{2}\right)^{2} /\left(q_{1}\right.\right.\right.$ $\left.\left.\mu_{2}+\mu_{1} \mu_{2}+q_{2} \mu_{1}\right)^{2}\right)-\left(\left(q_{1}^{2} \mu_{2}+q_{2}^{2} \mu_{1}\right)\left(q_{1}+q_{2}+\mu_{1}+\mu_{2}\right) /\left(q_{1} \mu_{2}\right.\right.$ $\left.\left.\left.+q_{2} \mu_{1}\right)\left(q_{1} \mu_{2}+\mu_{1} \mu_{2}+q_{2} \mu_{1}\right)^{2}\right)-\left(1 /\left(q_{1} \mu_{2}+\mu_{1} \mu_{2}+q_{2} \mu_{1}\right)\right)\right]$, respectively. The mean and variance of the number of customers served during a busy period when initiated by $m$ customers can be obtained from (14) and (23).

3.14. Interrupted Poisson Distribution. The interrupted Poisson distribution (IPP) is a special case of the two-state MMPP, described by the following two matrices:

$$
\begin{aligned}
& Q=\left(\begin{array}{cc}
-q_{1} & q_{1} \\
q_{2} & -q_{2}
\end{array}\right), \\
& M=\left(\begin{array}{ll}
\mu & 0 \\
0 & \mu
\end{array}\right) .
\end{aligned}
$$

The Laplace transform is given by $\bar{b}(s)=\left(\left(s+q_{2}\right) \mu\right) /$ $\left(s^{2}+\left(q_{1}+q_{2}+\mu\right) s+q_{2} \mu\right)$. An IPP is stochastically equivalent to a hyperexponential distribution [24]. The following parameter transformations relate the IPP to the hyperexponential distribution:

$$
\begin{aligned}
& \mathrm{IPP} \longrightarrow \mathrm{H}_{2} \\
& \mu_{1}=\frac{1}{2}\left\{\mu+q_{1}+q_{2}+\sqrt{\left(\mu+q_{1}+q_{2}\right)^{2}-4 \mu q_{2}}\right\}, \\
& \mu_{2}=\frac{1}{2}\left\{\mu+q_{1}+q_{2}-\sqrt{\left(\mu+q_{1}+q_{2}\right)^{2}-4 \mu q_{2}}\right\}, \\
& p_{1}=\frac{\mu-\mu_{2}}{\mu_{1}-\mu_{2}} \\
& p_{2}=1-p_{1} \text {. }
\end{aligned}
$$

Similarly, the following parameter transformations relate the hyperexponential to the IPP distribution: 
TABle 1: Distribution of the number of customers served during a busy period.

\begin{tabular}{|c|c|c|c|c|c|c|}
\hline \multirow{2}{*}{$n$} & \multicolumn{3}{|c|}{$\begin{array}{l}\text { Exponential distribution } \\
\rho=0.75, \lambda=0.2, \mu=0.8\end{array}$} & \multicolumn{3}{|c|}{$\begin{array}{c}\text { Deterministic distribution } \\
\rho=0.4, \lambda=0.2, k=2\end{array}$} \\
\hline & $\begin{array}{c}m=3 \\
P_{n}\end{array}$ & $\begin{array}{c}m=7 \\
P_{n}\end{array}$ & $\begin{array}{c}m=9 \\
P_{n}\end{array}$ & $\begin{array}{c}m=3 \\
P_{n}\end{array}$ & $\begin{array}{c}m=7 \\
P_{n}\end{array}$ & $\begin{array}{c}m=9 \\
P_{n}\end{array}$ \\
\hline 3 & 0.512000 & & & 0.301194 & & \\
\hline 4 & 0.245760 & & & 0.242276 & & \\
\hline 5 & 0.117965 & & & 0.162402 & & \\
\hline 6 & 0.058720 & & & 0.104507 & & \\
\hline 7 & 0.030199 & 0.209715 & & 0.066745 & 0.060810 & \\
\hline 8 & 0.015945 & 0.234881 & & 0.042742 & 0.114134 & \\
\hline 9 & 0.008599 & 0.187905 & 0.134218 & 0.027536 & 0.137712 & 0.027324 \\
\hline 10 & 0.004717 & 0.132285 & 0.193274 & 0.017862 & 0.136757 & 0.065936 \\
\hline 20 & 0.000019 & 0.001148 & 0.005804 & 0.000319 & 0.010366 & 0.032485 \\
\hline 30 & 0.000000 & 0.000009 & 0.000052 & 0.000008 & 0.000367 & 0.001660 \\
\hline$\vdots$ & $\vdots$ & $\vdots$ & $\vdots$ & $\vdots$ & $\vdots$ & $\vdots$ \\
\hline$E(N)$ & 4.000 & 9.3333 & 12.000 & 5.000 & 11.6667 & 15.000 \\
\hline$E\left(N^{2}\right)$ & 18.222 & 92.296 & 150.67 & 30.556 & 149.074 & 241.667 \\
\hline $\operatorname{Var}(N)$ & 2.2222 & 5.1852 & 6.6667 & 5.5556 & 12.963 & 16.6667 \\
\hline
\end{tabular}

TABle 2: Distribution of the number of customers served during a busy period.

\begin{tabular}{|c|c|c|c|c|c|c|}
\hline \multirow{2}{*}{$n$} & \multicolumn{3}{|c|}{$\begin{array}{l}\text { Generalized Erlang distribution } \\
\rho=0.75, \lambda=0.5, \mu_{1}=1, \mu_{2}=2\end{array}$} & \multicolumn{3}{|c|}{$\begin{array}{c}\text { Hyperexponential distribution } \\
\rho=0.4, \lambda=0.5, \mu_{1}=1, \mu_{2}=2, p_{1}=0.6, p_{2}=0.4\end{array}$} \\
\hline & $\begin{array}{c}m=3 \\
P_{n}\end{array}$ & $\begin{array}{c}m=7 \\
P_{n}\end{array}$ & $\begin{array}{c}m=9 \\
P_{n}\end{array}$ & $\begin{array}{c}m=3 \\
P_{n}\end{array}$ & $\begin{array}{c}m=7 \\
P_{n}\end{array}$ & $\begin{array}{c}m=9 \\
P_{n}\end{array}$ \\
\hline 3 & 0.151704 & & & 0.373248 & & \\
\hline 4 & 0.129454 & & & 0.220963 & & \\
\hline 5 & 0.101837 & & & 0.133358 & & \\
\hline 6 & 0.080573 & & & 0.084303 & & \\
\hline 7 & 0.064895 & 0.012274 & & 0.055373 & 0.100306 & \\
\hline 8 & 0.053219 & 0.024439 & & 0.037470 & 0.138556 & \\
\hline 9 & 0.044348 & 0.033129 & 0.003491 & 0.025955 & 0.138307 & 0.051999 \\
\hline 10 & 0.037468 & 0.038404 & 0.008938 & 0.018318 & 0.122466 & 0.092350 \\
\hline 20 & 0.010875 & 0.029860 & 0.030692 & 0.000971 & 0.012428 & 0.029950 \\
\hline 50 & 0.001310 & 0.005343 & 0.008297 & 0.000001 & 0.000014 & 0.000044 \\
\hline 100 & 0.000129 & 0.000588 & 0.001014 & 0.000000 & 0.000000 & 0.000000 \\
\hline 200 & 0.000003 & 0.000017 & 0.000030 & 0.000000 & 0.000000 & 0.000000 \\
\hline$\vdots$ & $\vdots$ & $\vdots$ & $\vdots$ & $\vdots$ & $\vdots$ & $\vdots$ \\
\hline$E(N)$ & 12.000 & 28.000 & 36.000 & 5.000 & 11.667 & 15.000 \\
\hline$E\left(N^{2}\right)$ & 348.00 & 1260.00 & 1908.00 & 33.194 & 155.231 & 249.583 \\
\hline $\operatorname{Var}(N)$ & 204.00 & 476.000 & 612.00 & 8.1944 & 19.1204 & 24.5833 \\
\hline
\end{tabular}

$$
\begin{aligned}
H_{2} & \longrightarrow \text { IPP, } \\
\mu & =p_{1} \mu_{1}+p_{2} \mu_{2}, \\
q_{1} & =\frac{p_{1} p_{2}\left(\mu_{1}-\mu_{2}\right)^{2}}{\mu}, \\
q_{2} & =\frac{\mu_{1} \mu_{2}}{\mu} .
\end{aligned}
$$

Substituting the LT of IPP service-time distribution $\bar{b}(s)$ in functional equation (2) and applying Lagrange's inversion theorem, we get the distribution of the number of customers served during a busy period when initiated by $m$ customers as follows:

$$
\begin{aligned}
P\left(N_{m}=n\right)= & \frac{m}{n} K_{1}^{n}\left(\lambda^{2} \psi_{1} \psi_{2}\right)^{-n} \sum_{i=0}^{n-m} \sum_{j=0}^{n-m-i} \\
& \cdot\left(\begin{array}{c}
2 n-m-i-j-1 \\
n-m-i-j
\end{array}\right)\left(\begin{array}{c}
n+i-1 \\
i
\end{array}\right) \\
& \times\left(\begin{array}{c}
n \\
j
\end{array}\right)(-1)^{j} \psi_{1}^{m+i+j-n} \psi_{2}^{-i} K_{2}^{j},
\end{aligned}
$$


Table 3: Distribution of the number of customers served during a busy period.

\begin{tabular}{|c|c|c|c|c|c|c|}
\hline \multirow{2}{*}{$n$} & \multicolumn{3}{|c|}{$\begin{array}{l}\text { Matrix exponential distribution } \\
\rho=0.42, \lambda=0.4, \mu=1.049\end{array}$} & \multicolumn{3}{|c|}{$\begin{array}{c}\text { Chi-square distribution } \\
\rho=0.6, \lambda=0.2, k=3\end{array}$} \\
\hline & $\begin{array}{c}m=3 \\
P_{n}\end{array}$ & $\begin{array}{c}m=7 \\
P_{n}\end{array}$ & $\begin{array}{c}m=9 \\
P_{n}\end{array}$ & $\begin{array}{c}m=3 \\
P_{n}\end{array}$ & $\begin{array}{c}m=7 \\
P_{n}\end{array}$ & $\begin{array}{c}m=9 \\
P_{n}\end{array}$ \\
\hline 3 & 0.339685 & & & 0.220001 & & \\
\hline 4 & 0.222371 & & & 0.170756 & & \\
\hline 5 & 0.139825 & & & 0.125171 & & \\
\hline 6 & 0.090430 & & & 0.093132 & & \\
\hline 7 & 0.060262 & 0.080509 & & 0.070848 & 0.029218 & \\
\hline 8 & 0.041179 & 0.122977 & & 0.055009 & 0.052916 & \\
\hline 9 & 0.028722 & 0.130997 & 0.039195 & 0.043465 & 0.066170 & 0.010648 \\
\hline 10 & 0.020371 & 0.121409 & 0.076975 & 0.034852 & 0.071365 & 0.024794 \\
\hline 20 & 0.001091 & 0.014350 & 0.033872 & 0.006110 & 0.031203 & 0.044522 \\
\hline 50 & 0.000001 & 0.000016 & 0.000054 & 0.000167 & 0.001229 & 0.002577 \\
\hline 100 & 0.000000 & 0.000000 & 0.000000 & 0.000001 & 0.000011 & 0.000026 \\
\hline$\vdots$ & $\vdots$ & $\vdots$ & $\vdots$ & $\vdots$ & $\vdots$ & $\vdots$ \\
\hline$\dot{E}(N)$ & 5.1703 & 12.0640 & 15.5109 & 7.5000 & 17.5000 & 22.5000 \\
\hline$E\left(N^{2}\right)$ & 35.5201 & 166.047 & 266.953 & 95.625 & 398.125 & 118.125 \\
\hline $\operatorname{Var}(N)$ & 8.7880 & 20.5054 & 26.3641 & 39.375 & 91.875 & 624.375 \\
\hline
\end{tabular}

TABle 4: Distribution of the number of customers served during a busy period.

\begin{tabular}{|c|c|c|c|c|c|c|}
\hline \multirow[t]{2}{*}{$n$} & $\lambda=0.5, \mu_{1}$ & $\begin{array}{c}\text { type distri } \\
1, r_{10}=1 \\
r_{21}=0\end{array}$ & $3, r_{20}=1$ & $\lambda=0.5$ & $\mu_{2}=2, p_{1}$ & $=0.25$ \\
\hline & $\begin{array}{c}m=3 \\
P_{n}\end{array}$ & $\begin{array}{c}m=7 \\
P_{n}\end{array}$ & $\begin{array}{c}m=9 \\
P_{n}\end{array}$ & $\begin{array}{c}m=3 \\
P_{n}\end{array}$ & $\begin{array}{c}m=7 \\
P_{n}\end{array}$ & $\begin{array}{c}m=9 \\
P_{n}\end{array}$ \\
\hline 3 & 0.296296 & & & 0.361705 & & \\
\hline 4 & 0.197531 & & & 0.225840 & & \\
\hline 5 & 0.131687 & & & 0.138872 & & \\
\hline 6 & 0.091043 & & & 0.087950 & & \\
\hline 7 & 0.065031 & 0.058528 & & 0.057338 & 0.093217 & \\
\hline 8 & 0.047689 & 0.091043 & & 0.038294 & 0.135805 & \\
\hline 9 & 0.035718 & 0.101159 & 0.026012 & 0.026084 & 0.140038 & 0.047322 \\
\hline 10 & 0.027214 & 0.098911 & 0.052025 & 0.018057 & 0.126330 & 0.088640 \\
\hline 20 & 0.003003 & 0.022933 & 0.041731 & 0.000749 & 0.011701 & 0.029999 \\
\hline 50 & 0.000022 & 0.000226 & 0.000565 & 0.000000 & 0.000006 & 0.000022 \\
\hline$\vdots$ & $\vdots$ & $\vdots$ & $\vdots$ & $\vdots$ & $\vdots$ & $\vdots$ \\
\hline$E(N)$ & 6.000 & 14.000 & 18.000 & 4.9660 & 11.586 & 14.897 \\
\hline$E\left(N^{2}\right)$ & 54.000 & 238.00 & 378.00 & 31.925 & 151.20 & 243.71 \\
\hline $\operatorname{Var}(N)$ & 18.000 & 42.000 & 54.000 & 7.2680 & 16.959 & 21.805 \\
\hline
\end{tabular}

where

$$
\begin{aligned}
\psi_{1,2} & =\frac{\left(q_{1}+q_{2}+\mu+2 \lambda\right) \pm \sqrt{\left(q_{1}+q_{2}+\mu\right)^{2}-4 q_{2} \mu}}{2 \lambda} \\
K_{1} & =q_{2} \mu^{2}\left(\lambda+q_{2}\right), \\
K_{2} & =\frac{\lambda}{\lambda+q_{2}} .
\end{aligned}
$$

The mean, second moment, and variance of the IPP distribution are $\nu_{1}=\left(q_{1}+q_{2}\right) / \mu q_{2}, \nu_{2}=2\left[\mu q_{1}+\left(q_{1}+q_{2}\right)^{2}\right] /$ $\left(\mu q_{2}\right)^{2}$, and $\sigma^{2}=\left(2 \mu q_{1}+\left(q_{1}+q_{2}\right)^{2}\right) /\left(\mu q_{2}\right)^{2}$, respectively. The mean and variance of the distribution of the number of customers served during a busy period when initiated by $m$ customers can be obtained from (14) and (15). The mean and variance of the number of customers served during a busy period can be obtained as

$$
\begin{aligned}
E\left(N_{m}\right) & =\frac{m \mu q_{2}}{\mu q_{2}-\lambda\left(q_{1}+q_{2}\right)}, \\
\operatorname{Var}\left(N_{m}\right) & =\frac{m \lambda \mu q_{2}\left(\left(q_{1}+q_{2}\right)\left(\mu q_{2}+\lambda\left(q_{1}+q_{2}\right)\right)+2 \lambda \mu q_{1}\right)}{\left(\mu q_{2}-\lambda\left(q_{1}+q_{2}\right)\right)^{3}} .
\end{aligned}
$$

\section{Numerical Results}

In this section, we present numerical results, for a few cases, for the distribution of the number served during a busy period when initiated by $m$ customers in Tables $1-5$. We 
TABle 5: Distribution of the number of customers served during a busy period.

\begin{tabular}{|c|c|c|c|c|c|c|}
\hline \multirow[t]{2}{*}{$n$} & \multicolumn{3}{|c|}{$\begin{array}{l}\text { Markov-modulated Poisson process } \\
\qquad \begin{array}{c}\rho=0.214, \lambda=0.5, \mu_{1}=5 \\
\mu_{2}=1, r_{1}=1, r_{2}=0.5\end{array}\end{array}$} & \multicolumn{3}{|c|}{$\begin{array}{l}\text { Interrupted Poisson process } \\
\qquad \begin{array}{c}\rho=0.35, \lambda=0.5, \mu_{1}=2 \\
\mu_{2}=1, r_{1}=0.15, r_{2}=1.25\end{array}\end{array}$} \\
\hline & $\begin{array}{c}m=3 \\
P_{n}\end{array}$ & $\begin{array}{c}m=7 \\
P_{n}\end{array}$ & $\begin{array}{c}m=9 \\
P_{n}\end{array}$ & $\begin{array}{c}m=3 \\
P_{n}\end{array}$ & $\begin{array}{c}m=7 \\
P_{n}\end{array}$ & $\begin{array}{c}m=9 \\
P_{n}\end{array}$ \\
\hline 3 & 0.580690 & & & 0.416275 & & \\
\hline 4 & 0.225374 & & & 0.230894 & & \\
\hline 5 & 0.097992 & & & 0.130911 & & \\
\hline 6 & 0.046357 & & & 0.077951 & & \\
\hline 7 & 0.023164 & 0.281322 & & 0.048328 & 0.129386 & \\
\hline 8 & 0.012020 & 0.254765 & & 0.030916 & 0.167454 & \\
\hline 9 & 0.006413 & 0.176690 & 0.195809 & 0.020268 & 0.156863 & 0.072134 \\
\hline 10 & 0.003494 & 0.112568 & 0.227989 & 0.013549 & 0.130565 & 0.120031 \\
\hline 20 & 0.000015 & 0.000724 & 0.003611 & 0.000426 & 0.007541 & 0.021595 \\
\hline 50 & 0.000000 & 0.000000 & 0.000000 & 0.000000 & 0.000002 & 0.000007 \\
\hline$\vdots$ & $\vdots$ & $\vdots$ & $\vdots$ & $\vdots$ & $\vdots$ & $\vdots$ \\
\hline$E(N)$ & 3.81818 & 8.9091 & 11.4545 & 4.6154 & 10.7692 & 13.8462 \\
\hline$E\left(N^{2}\right)$ & 16.4254 & 83.681 & 136.747 & 26.7911 & 128.785 & 208.184 \\
\hline $\operatorname{Var}(N)$ & 1.8469 & 4.3095 & 5.5407 & 5.4893 & 12.8084 & 16.4679 \\
\hline
\end{tabular}

consider that service-times are distributed according to an exponential, deterministic, generalized Erlang, hyperexponential, matrix exponential, chi-square, phase-type, Coxian, Markov-modulated Poisson process, and interrupted Poisson process. The mean and variance of the number of customers served during a busy period were calculated using both the distributions as well as the corresponding formulas. The matching of the results was excellent. The behavior of the probability distribution is basically the same for various service-time distributions. The most frequent number of customers served during the busy period is when initiated with less number of customers. The mean and variance of the number of customers served during a busy period increase with the increase of $m$, where $m$ is the number that initiates the busy period.

\section{Conclusion}

The analysis of the distribution of the number of customers served during a busy period is important as it helps us to study the traffic intensity and the cycle time of the queueing system. In this paper, we have developed the analytically explicit results for the distribution of the number of customers served during a busy period for special cases of the $M / G / 1$ queues. The functional equation for the Laplace transform of the number of customers served during a busy period for the $M / G / 1$ queue is widely known, but several researchers state that, in general, it is not easy to invert it except for some simple cases such $M / M / 1$ and $M / D / 1$. We present an elegant solution to determine the distribution of the number of customers served during a busy period and the computation of distributions as well as their moments using the Lagrange inversion theorem and MAPLE. We obtain the distribution of the number of customers served during a busy period for various service-time distributions, such as exponential, deterministic, Erlang- $k$, gamma, chisquare, inverse Gaussian, generalized Erlang, matrix exponential, hyperexponential, uniform, Coxian, phasetype, Markov-modulated Poisson process, and interrupted Poisson process. We have also presented computational results using our method. The associated derivations are very fast and robust due to the lucidity of the expressions. Using this approach, the method can be applied to other servicetime distributions such as Weibull and Gaussian.

\section{Data Availability}

No data were used to support this study.

\section{Conflicts of Interest}

The authors declare that they have no conflicts of interest.

\section{Acknowledgments}

This research was partially supported by NSERC under research grant number RGPIN-2014-06604.

\section{References}

[1] J. Abate and W. Whitt, "The Fourier-series method for inverting transforms of probability distributions," Queueing Systems, vol. 10, no. 1-2, pp. 5-87, 1992.

[2] J. Abate and W. Whitt, "Numerical inversion of probability generating functions," Operations Research Letters, vol. 12, no. 4, pp. 245-251, 1992.

[3] J. Abate and W. Whitt, "Solving probability transform functional equations for numerical inversion," Operations Research Letters, vol. 12, no. 5, pp. 275-281, 1992.

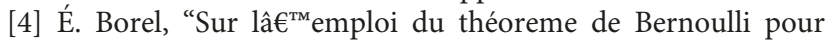
faciliter le calcul dâ $€^{\mathrm{TM}}$ une infinité de coefficients: application au probleme de lâ $€^{\mathrm{TM}}$ attentea un guichet," Comptes rendus de l'Académie des Sciences, vol. 214, pp. 452-456, 1942.

[5] J. C. Tanner, "A problem of interference between two queues," Biometrika, vol. 40, no. 1-2, pp. 58-69, 1953. 
[6] D. G. Kendall, "Some problems in the theory of dams," Journal of the Royal Statistical Society: Series B (Methodological), vol. 19, no. 2, pp. 207-212, 1957.

[7] F. A. Haight, "A distribution analogous to the Borel-Tanner," Biometrika, vol. 48, no. 1-2, pp. 167-173, 1961.

[8] N. U. Prabhu, "Some results for the queue with poisson arrivals," Journal of the Royal Statistical Society: Series B (Methodological), vol. 22, no. 1, pp. 104-107, 1960.

[9] N. U. Prabhu, Queues and Inventories, John Wiley \& Sons, Hoboken, NJ, USA, 1965.

[10] F. A. Haight, Mathematical Theories of Traffic Flow, Vol. 7, Academic Press, Cambridge, MA, USA, 1963.

[11] F. A. Haight and M. A. Breuer, "The Borel-Tanner distribution," Biometrika, vol. 47, no. 1-2, pp. 143-150, 1960.

[12] L. Takács, "Investigation of waiting time problems by reduction to Markov processes," Acta Mathematica Hungarica, vol. 6, no. 1-2, pp. 101-129, 1955.

[13] L. Takacs, "A generalization of the ballot problem and its application in the theory of queues," Journal of the American Statistical Association, vol. 57, no. 298, pp. 327-337, 1962.

[14] A. W. Kemp and C. D. Kemp, "On a distribution associated with certain stochastic processes," Journal of the Royal Statistical Society: Series B (Methodological), vol. 30, no. 1, pp. 160-163, 1968.

[15] W. Feller, An Introduction to Probability Theory and Its Applications, Vol. 2, John Wiley \& Sons, Hoboken, NJ, USA, 2008.

[16] L. Takács, On Combinatorial Methods in the Theory of Stochastic Processes, Technical report, Columbia University, New York, USA, 1967.

[17] D. P. Heyman, "An approximation for the busy period of the M/G/1 queue using a diffusion model," Journal of Applied Probability, vol. 11, no. 1, pp. 159-169, 1974.

[18] P. C. Consul and L. R. Shenton, "Use of Lagrange expansion for generating discrete generalized probability distributions," SIAM Journal on Applied Mathematics, vol. 23, no. 2, pp. 239-248, 1972.

[19] L. Kleinrock, Queueing Systems: Theory, Vol. 1, John Wiley \& Sons, Inc., New York, USA, 1975.

[20] J. Medhi, Stochastic Models in Queueing Theory, Elsevier, Amsterdam, Netherlands, 2002.

[21] W. J. Stewart, Probability, Markov Chains, Queues, and Simulation: The Mathematical Basis of Performance Modeling, Princeton University Press, Princeton, NJ, USA, 2009.

[22] P. C. Consul and F. Famoye, Lagrangian Probability Distributions, Springer, Berlin, Germany, 2006.

[23] J. L. Jain, S. G. Mohanty, and W. Böhm, A Course on Queueing Models, Chapman and Hall/CRC Press, Boca Raton, FL, USA, 2016.

[24] W. Fischer and K. Meier-Hellstern, "The Markov-modulated poisson process (MMPP) cookbook," Performance Evaluation, vol. 18, no. 2, pp. 149-171, 1993. 


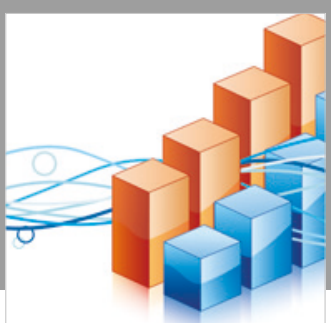

Advances in

Operations Research

\section{-n-m}
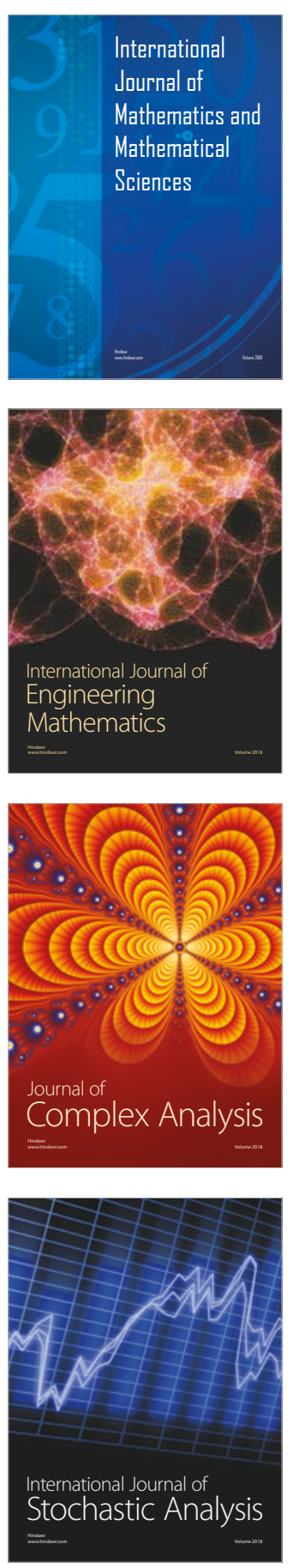
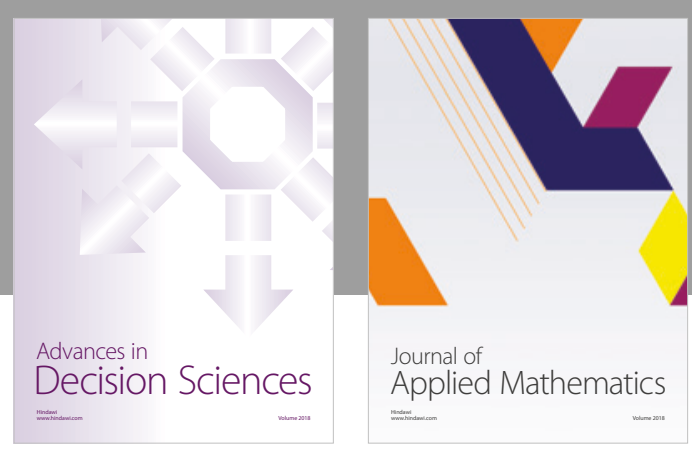

Journal of

Applied Mathematics
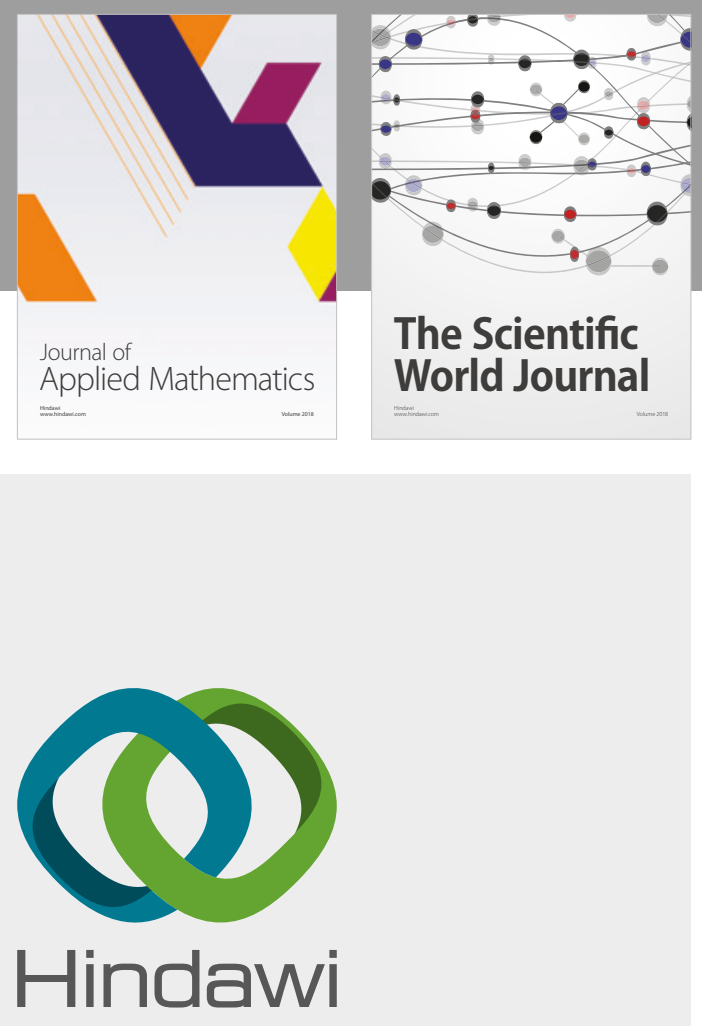

Submit your manuscripts at

www.hindawi.com

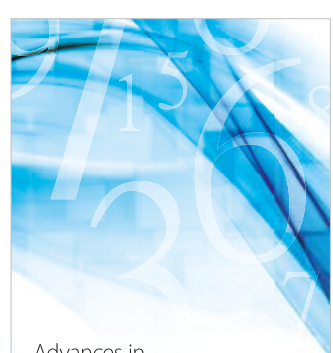

Advances in
Numerical Analysis
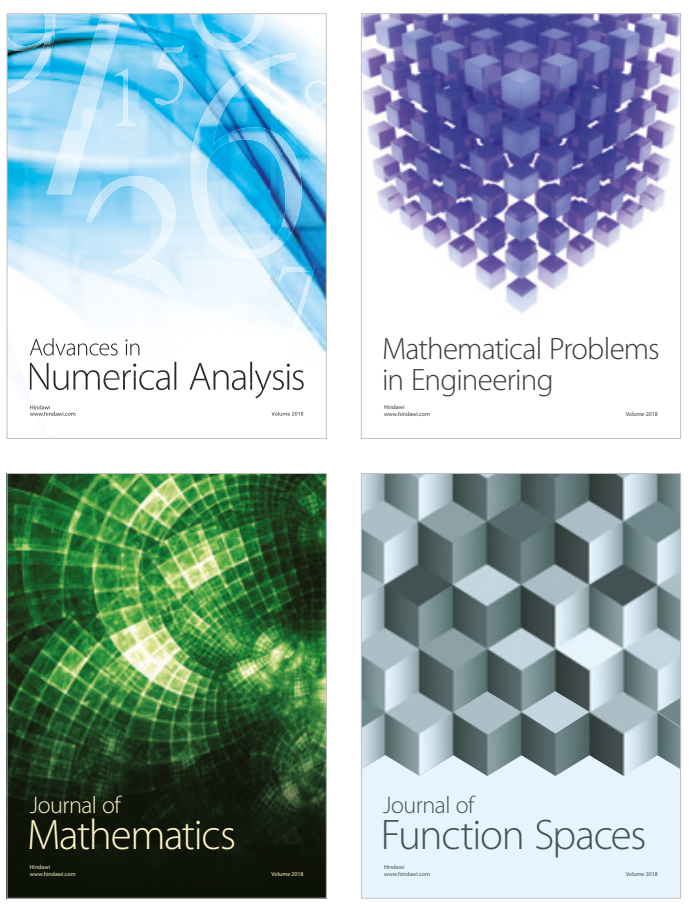

Mathematical Problems in Engineering

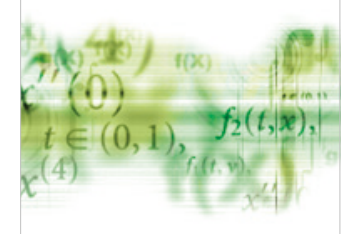

International Journal of

Differential Equations

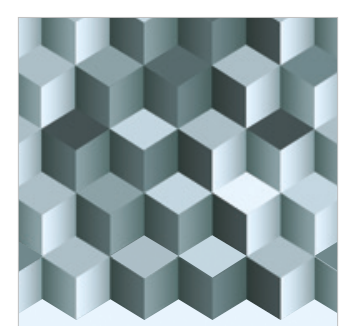

Journal of

Function Spaces

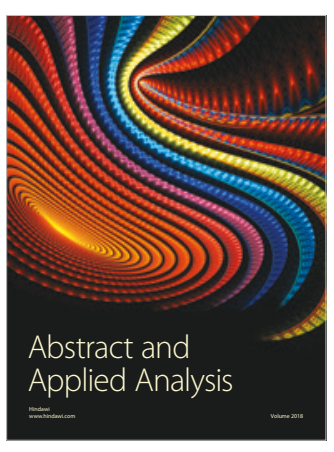

The Scientific

World Journal

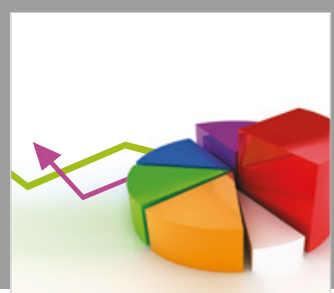

Journal of

Probability and Statistics
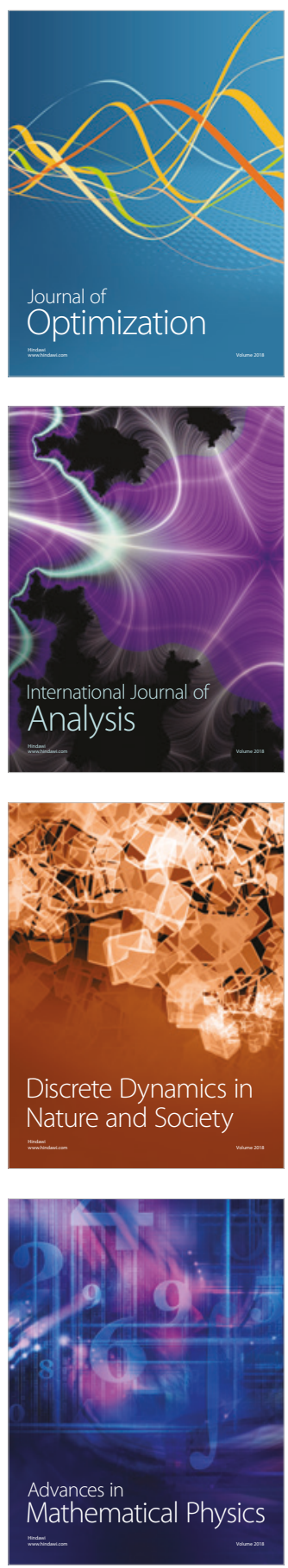